Acta Cryst. (2002). A58 (Supplement), C53

STRUCTURAL RELAXATION BETWEEN HIGH-DENSITY AND

LOW-DENSITY AMORPHOUS ICE: AN X-RAY AND NEUTRON DIFFRACTION STUDY

$\begin{array}{lllll}\text { C. A. Tulk } & 1 \\ \text { C. J. Benmore } & \text { D. D. Klug } & \text { J. Uquirdi } & \text { B. Tomberli } & \text { J. }\end{array}$ Neuefeind $^{2}$ P. A. Egelstaff ${ }^{4}$

${ }^{1}$ Oak Ridge National Laboratory, Oak Ridge, TN USA $3783{ }^{2}$ Argonne National Laboratory, Argonne IL USA $6043{ }^{3}$ National Research Council of Canada, Ottawa ON Canada K1A 0R ${ }^{4}$ Department of Physics, University of Guelph, Guelph ON Canada N1G 2W

High-density amorphous ice, prepared by compression of ice I, has been annealed isothermally for several hours at 95, 100, 105 and $115 \mathrm{k}$ and structural changes have been continuously monitored using synchrotron $\mathrm{x}$-ray and neutron diffraction techniques. During each anneal structural changes are indicated by changes in the first sharp diffraction peak position in the static structure factor. During each anneal the peak position is initially observed to rapidly change position toward the LDA phase, however, at each anneal temperature the transformation is observed to stop before completion of the HDA to LDA transition, resulting in several metastable intermediate phases. The structure of these intermediate phases were then temperature quenched by rapidly cooling the sample to $40 \mathrm{k}$, thus allowing detailed $\mathrm{S}(\mathrm{Q})$ and $\mathrm{G}(\mathrm{r})$ functions to be determined. It is shown that each intermediate phase is unique and the static structure factor functions cannot be reproduced by any linear combination of the measured $\mathrm{s}(\mathrm{Q})$ of HDA and LDA. These studies also represent the first comparison of data collected using synchrotron X-ray and neutron diffraction from subsections of the same sample. Using this technique we have quench recovered a total of three metastable intermediate phases, with the implication that there are and infinite number of possible states, thus indicating that the HDA to LDA transition at zero pressure is much more complicated than first thought.

Keywords: AMORPHOUS, ICE, DIFFRACTION

Acta Cryst. (2002). A58 (Supplement), C53

STRUCTURE OF PERMANENTLY DENSIFIED GeO $\mathrm{GLASS}_{2}$ $\underline{\text { S. Sampath }}^{1,2}$ C.J. Benmore ${ }^{1}$ D.L. Price ${ }^{3}$ J. Neuefeind ${ }^{4}$ J. Yarger ${ }^{2}$ ${ }_{1}^{1}$ I.P.N.S., Argonne National Labaratory, IL - 60439 USA. ${ }^{2}$ Dept. of Chemistry, University of Wyoming, Laramie, WY - $8207{ }^{3}$ M.S.D., Argonne National Labaratory, IL - 60439. ${ }^{4}$ Chemistry division, Argonne National Labaratory, IL - 60439.

Pressure induced amorphous transformations are known to occur in several tetrahedral systems and can result in permanently densified glasses (PDG) upon release of the pressure. In-situ studies on $\mathrm{v}-\mathrm{SiO}_{2}$ and $\mathrm{v}-\mathrm{GeO}_{2}$ indicate that there is a reversible change in the cation coordination with pressure. In PDG, evidences of an irreversible effect suggest retention of the high-pressure phase. We report a combination of pulsed neutron diffraction and high-energy x-ray diffraction measurements on normal and densified $\mathrm{GeO}_{2}(10 \mathrm{GPa})$ from which information on all the three nearest neighbor (NN) correlations have been obtained. Our results elucidate structural changes both in the short and intermediate range order ( $\mathrm{SRO}$ and IRO) of $\mathrm{GeO}_{2}$ glass. Pressurization results in a reduction in the inter-tetrahedral Ge-O-Ge and the intra-tetrahedral O-Ge$\mathrm{O}$ angles. This frustration caused is accommodated as a decrease in the nextnear neighbor (NNN) Ge-OII and O-OII distances and an increase in the Ge-OI and $\mathrm{O}-\mathrm{OI}$ and Ge-Ge NN distances. Structural changes induced by densification, are brought about primarily by the reconfiguration in the NNN oxygen correlations, thereby reducing the IRO. This leads to a bending of the Ge-O-Ge angle and a subsequent distortion of the $\mathrm{GeO}_{4}$ tetrahedra. We propose that it is the NNN O-OII packing that determines the high-pressure structure of the $\mathrm{GeO}_{2}$ glass.

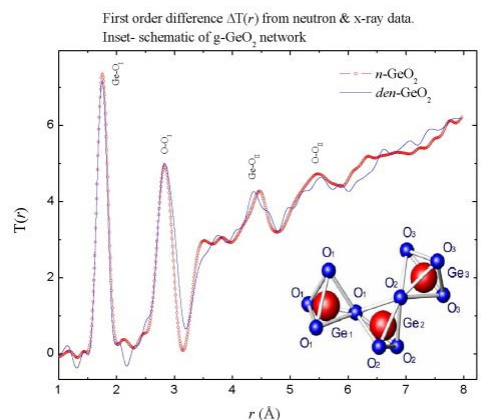

Keywords: GLASS, DENSIFICATION, DIFFRACTION
Acta Cryst. (2002). A58 (Supplement), C53

STRUCTURE OF HDA UNDER PRESSURE BY IN-SITU NEUTRON DIFFRACTION TO 2.5 GPa

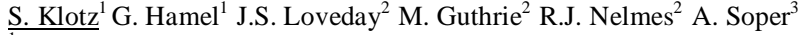

${ }^{1}$ Physique des Milieux Condenses, University P\&M Curie, B77, 75252 Paris, France ${ }^{2}$ Department of Physics and Astronomy and Centre of Science at Extreme Conditions, The University of Edinburgh, Edinburgh EH9 3JZ, UK ${ }^{3}$ The ISIS Facility, Rutherford Appleton Laboratory, Chilton, Didcot OX11 OQX, UK

When ordinary ice Ih is compressed to $1.5 \mathrm{GPa}$ at $77 \mathrm{~K}$ it transforms to highdensity amorphous ice (HDA). This form of ice has been extensively studied on samples recovered (decompressed) to ambient pressure, but little is known about its structure under pressure. Here we present the first in-situ diffraction studies of both the structural pressure dependence and the recrystallization behavior of HDA, extending over its entire range of existence of 0-2.5 GPa. We show that reliable structure factor data can be obtained by neutron diffraction on $100 \mathrm{~mm}^{3}$ samples of HDA under pressure in the ParisEdinburgh cell. A Reverse Monte Carlo analysis of the pattern allows extraction of all three partial correlation functions simultaneously. This analysis reveals that the compression in HDA is achieved by a partial collapse of the second coordination shell, which leads to a continuous increase of coordination from 4 to 6 , in a similar way that has been observed previously in high-density liquid water (HDW). On warming, HDA converts to ices III, IV, $\mathrm{V}$, VI and XII between 0.3 and $1.5 \mathrm{GPa}$. These phases are, without exception, the same as formed from liquid water along the melting line. These results seem to indicate that HDA is structurally very similar to high-density liquid water.

Keywords: HDA, STRUCTURE, PRESSURE
Acta Cryst. (2002). A58 (Supplement), C53

\section{THE STRUCTURE OF NORMAL AND SUPERCRITICAL BORON} TRIFLUORIDE AND RELATED STUDIES

J.F.C. Turner ${ }^{1}$ S.E McLain ${ }^{2,3}$ C.J. Benmore ${ }^{2}$ J. Urquidi $^{2}$

${ }^{1}$ Neutron Sciences Consortium and Department of Chemistry, University of Tennessee, Knoxville, Tennessee, USA ${ }^{2}$ IPNS, Argonne National Laboratory, S. Cass Avenue, Argonne, Illinois, USA ${ }^{3}$ Department of Chemistry, University of Tennessee, Knoxville, Tennessee,

The structure of normal phase and supercritical ${ }^{11} \mathrm{BF}_{3}$ has been investigated using neutron diffraction. The results of structural models for the normal fluid and the supercritical fluid are presented. The relation between the Lewis acidity and the structure is discussed. Comparisons are made with recent structural studies of superacidic fluids.

\section{Keywords: SUPERCITICAL FLUID STRUCTURE}

